\title{
Air Traffic Data Integration using the Semantic Web Approach
}

\author{
By Marcin Klauza* \\ Piotr Czekalski ${ }^{\dagger}$ \\ Krzysztof Tokarz:
}

Air traffic management systems require constant development. Whenever regular radar devices are out of range, the wide network of the receivers may constitute global air traffic monitoring solutions. Surveillance methods of controlling aircrafts are being improved and the one which stands apart from the others is $A D S-B$ (Automatic Dependent Surveillance Broadcast) introduced in most commercial and private aircrafts, and which was obligatory after the year 2020. Nowadays ADS-B receivers cover about $70 \%$ of European and 30\% of U.S air traffic. The ADB-S system is based on GPS communication. Aircrafts estimate their position using satellite based navigation systems. Along with plane position, there is a vast number of additional data broadcasted by the ADS-B transponder, including speed, altitude, plane and flight identification data, also emergency codes. The large amount of professional and amateur $A D S-B$ receivers located on most continents, covering significant amount of the airspace, has led to the conclusion that this kind of crowd-processing may establish valuable and reliable source of data using common interface. Currently there is no uniform layer of the $A D S-B$ data presentation and interfaces over the Web.

This paper regards standardisation of the data layer using WEB 3.0 - Semantic Web principals. It covers acquisition, processing and presentation of the data coming from the ADS-B receiver. A method of unifying data from distributed virtual radar stations has been proposed and is being presented in a way that allows this data to be combined across many sources with existing knowledge. Having $A D S-B$ information integrated and expressed in RDF (Resources Description Format), it would be easy to perform such a query against these data sets, using Protocol and RDF Query Language (SPARQL).

Now we stand at the verge of WEB 3.0, where applications vastly utilising Artificial Intelligence, semantic solutions and Natural Language Processing systems are going to become common.

${ }^{*}$ Student, Silesian University of Technology, Poland.

${ }^{\dagger}$ Assistant Professor, Silesian University of Technology, Poland.

${ }^{\star}$ Assistant Professor, Silesian University of Technology, Poland. 


\section{Introduction}

Air traffic management systems require constant development. Whenever regular radar devices are out of range, the wide network of the receivers may constitute global air traffic monitoring solutions. Surveillance methods of controlling aircrafts are being improved and the one which stands apart from the others is ADS-B (Automatic Dependent Surveillance Broadcast) introduced in most commercial and private aircrafts, obligatory after year 2020. The ADB$S$ system is based on GPS communication. Aircrafts estimate their position using satellite based navigation systems. Along with plane position, there is a vast number of additional data broadcasted by the ADS-B transponder, including speed, altitude, plane and flight identification data, and also emergency codes. However this data, coming from different sources has to be consolidated in order to be truly useful. In subsequent sections, the authors describe ADS-B hardware, its receiver-related data format and most important - a uniform layer solution based on Semantic Web concept.

\section{ADSB Receiver}

Figure 1. ADS-B System Design

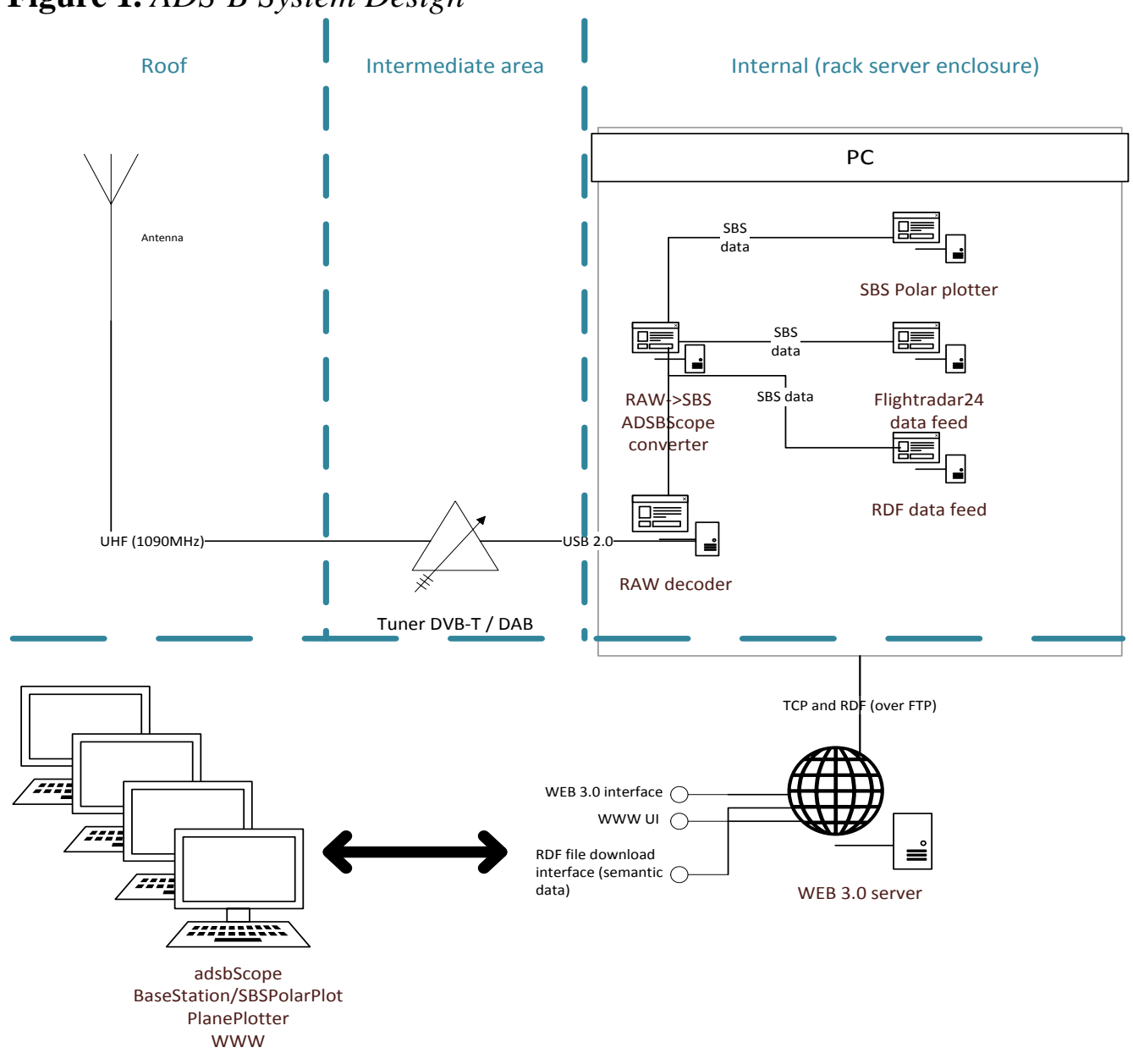


The ADSB receiver is an essential and core component of the system. A worldwide network of the receivers, capable to cover airspace may play a significant role in ensuring the security and safety of the air traffic. Most of it can be done with relatively small expenditure. One may find the wide number of the samples over the WEB, presenting solutions to the receiver antennas, high frequency down-samplers, protocol decoders, including those implemented as embedded devices (i.e. PIC based, see adsbPIC project by Sprut (2010)). The system components and connections between them are presented on Figure 1. It includes full receiver infrastructure, but its description is above the scope of this paper.

\section{The Antenna HF Section}

The first part of the data reception is the high frequency antenna. As noted by Balara (2010), it should be capable to receive communicates on $1090 \mathrm{MHz}$ frequency where all ADS-B communicates are transmitted. Because the HF receiver is usually connected to the antenna using a somewhat long cable (authors use about $7 \mathrm{~m}$ ) the antenna construction should bring the maximum possible gain to compensate signal drop. As the frequency of the ADS-B communicates is $1.09 \mathrm{GHz}$, the wavelength $\lambda$ in the copper collinear cable is given by:

$$
\lambda=\frac{k \cdot c}{f}
$$

where $c$-stands for the velocity of the light, $f=1090 \mathrm{MHz}, k=0.83$-velocity coefficient, for the RG-6U cable. The antenna is composed of 8 half $\lambda$ segments, connected such that the core of the first is connected to the shield of another and so on, constituting a chain by turns. There are two extra quarter $\lambda$ segments at each end of the antenna, improving its performance (Figure 2).

Figure 2. $A D S-B$ Coaxial Antenna

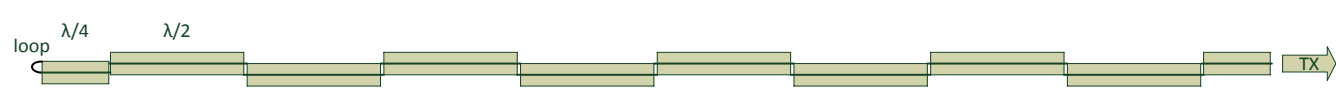

The antenna is located on the roof of the Faculty of Automation Control, Electronics and Computer Science building, about 40m over the ground level (Figure 3). The location of the antenna is N 50.288307019, E 18.67676767292. 
Figure 3. ADS-B Antenna Location

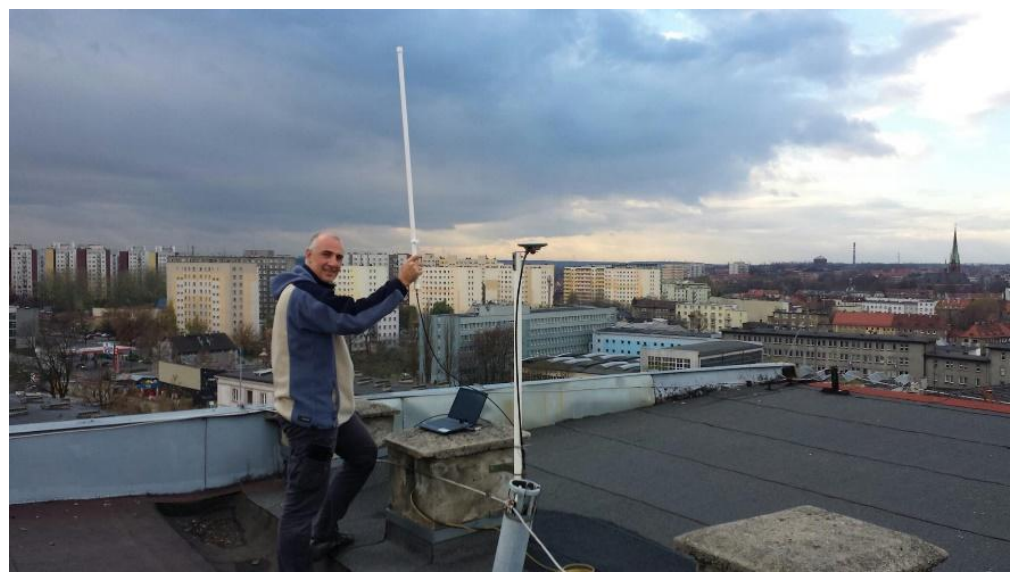

The range of the antenna is presented by the polar plot, based on the collection of over 13 million samples (received ADS-B communicates) within 6 months of work. The detected antenna range is $235 \mathrm{NM}$ radius max, the maximum altitude observed is 38275 feet, see Figure 4. The maximum amount of the planes observed simultaneously was over 200 and the average reception quality (measured by the number of the invalid frames) is about $80 \%$ subject to the weather changes and neighbor radio noise.

\section{Figure 4. Reception Range}

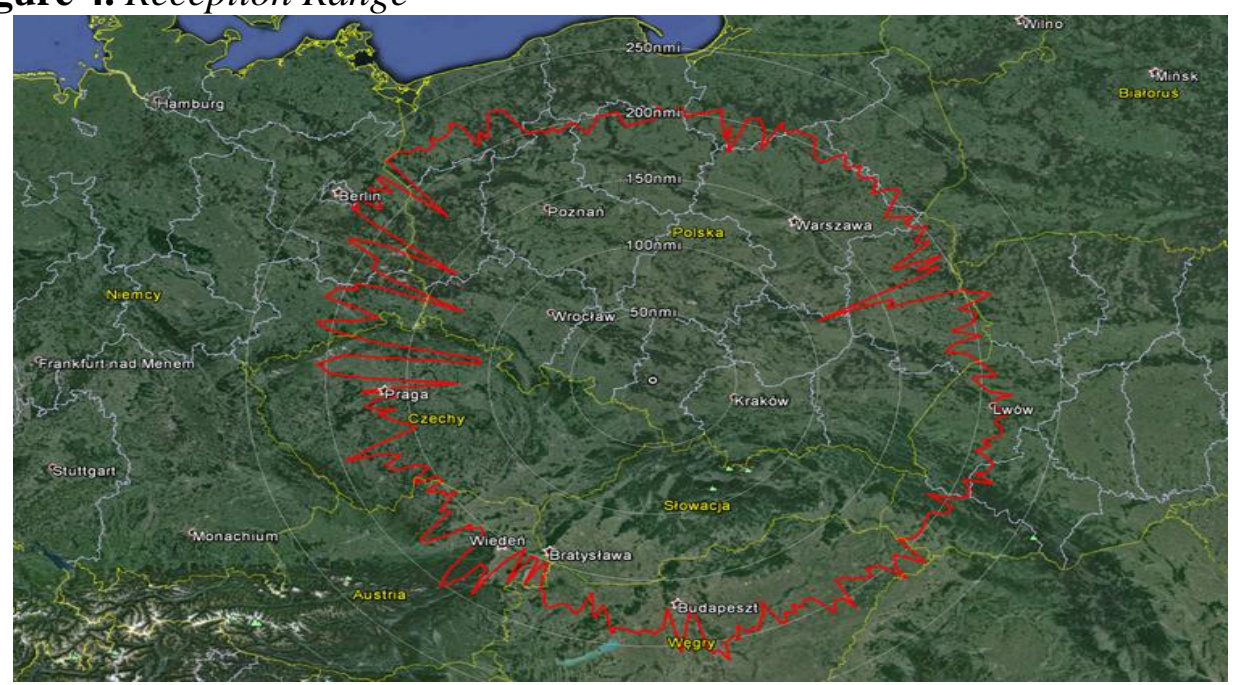

\section{The Receiver and Decoder Section}

The approach used by the authors of the paper was pretty straightforward an SDR $1^{1}$ approach to the ADS-B reception was implemented. It limits the implementation of the $\mathrm{HF}$ and decoder hardware to employing the appropriate

\footnotetext{
${ }^{1}$ SDR - Software Defined Radio - a software solution that is decoding the digital data that comes out as a stream (sound, vision, other) from the universal, programmable, wide range RF receiver, i.e. Radio or DVB-T USB dongle, connected to the PC computer (http://sdrradio.com/)
} 
DVB-T USB stick (Realtek RTL2832U and R820T tuner) acting as HF receiver / decoder as noted by Sprut (2010). The popular SDR ADSB\# software to decode ADS-B communicates was used (Touil, 2012). The ADSB\# decoder uses RAW (binary) data on its output, providing TCP/IP port. The adsbSCOPE software is a first line visualization and reporting solution that connects directly to the ADSB\# decoder using TCP, here via local loopback (Figure 1). It also contains an SBS (text based, BaseStation format) server, thus acting as a RAW to SBS converter. Authors use SBS data format, as presented in the following chapter.

\section{ADSB Data in Depth}

The SBS server provides data for programs connected to its TCP 30003 port, on the data stream basis. It is served in the BaseStation format, ASCII encoded, comprising information about aircrafts. Incoming data is divided into individual messages, each separated with new line characters (Figure 5). A Single message carries information coming either from aircraft or from a surveillance system. However, an individual message does not provide complete evidence of an aircraft, rather a piece of information, not necessarily being complete. Software has been developed for this reason and its role is to gather these scattered data, assemble them and finally present them to the user. This has been exhaustingly described in the subsequent section.

\section{Figure 5. Exemplary Data Stream Consisting of Individual Messages}

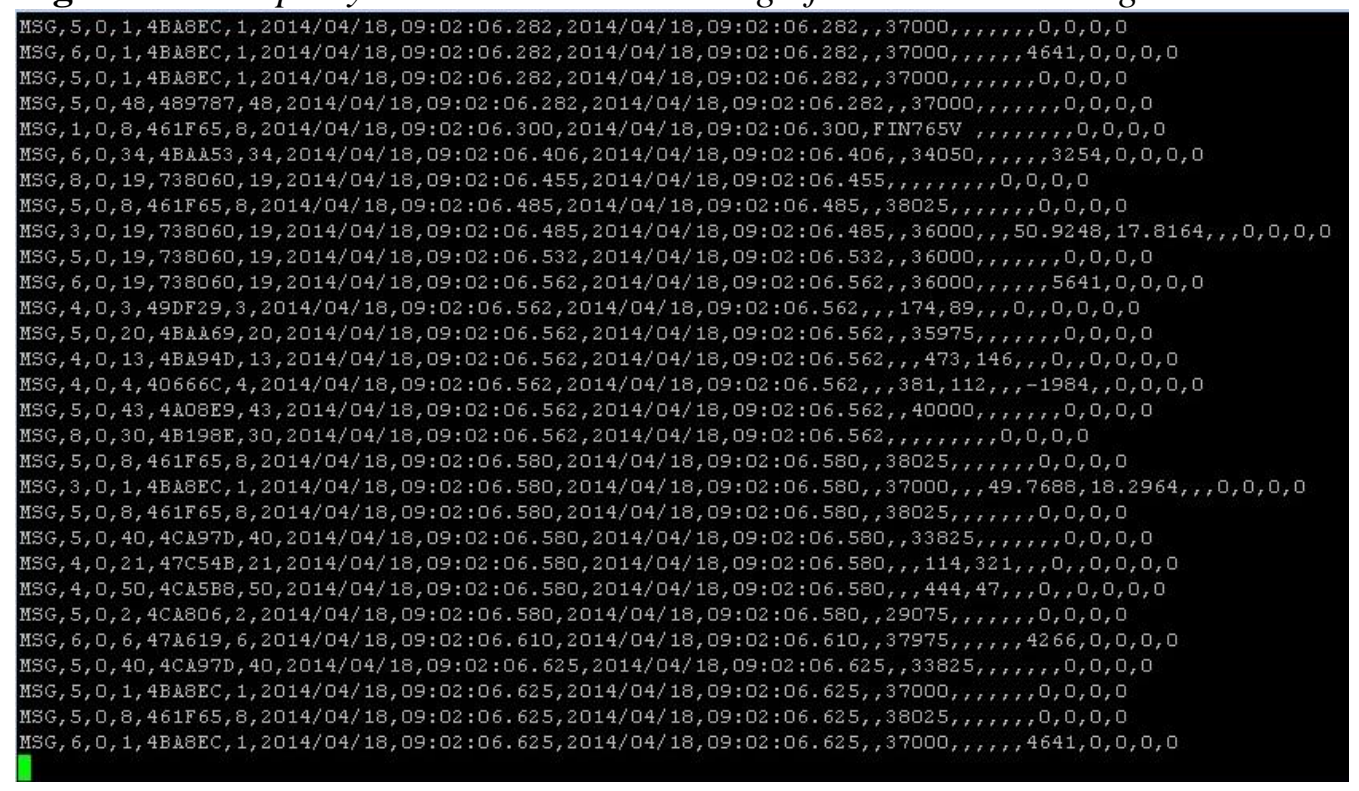

As Woodside (2013) concludes, on the top of BaseStation format there are six main types of messages (Table 1). The first five types come from the surveillance system, whereas $M S G$ is related to the messages generated by the aircraft hardware. 
Vol. 2, No. 2 Klauza et al.: Air Traffic Data Integration using the Semantic Web Approach

Table 1. Main Message Types (Woodside, 2013)

\begin{tabular}{|c|c|c|}
\hline ID & Type & Description \\
\hline SEL & $\begin{array}{c}\text { SELECTION CHANGE } \\
\text { MESSAGE }\end{array}$ & $\begin{array}{c}\text { Produced when the user changes the } \\
\text { selected aircraft in BaseStation. }\end{array}$ \\
\hline ID & NEW ID MESSAGE & $\begin{array}{c}\text { Produced when an aircraft being tracked } \\
\text { changes its callsign. }\end{array}$ \\
\hline AIR & NEW AIRCRAFT MESSAGE & $\begin{array}{c}\text { Produced when the SBS receives a new } \\
\text { signal for an aircraft that wasn't tracked so } \\
\text { far. }\end{array}$ \\
\hline STA & $\begin{array}{c}\text { SATUS CHANGE } \\
\text { MESSAGE }\end{array}$ & $\begin{array}{c}\text { Produced when an aircraft's status changes } \\
\text { due to the time-out values. }\end{array}$ \\
\hline MSK & CLICK MESSAGE & $\begin{array}{c}\text { Produced when the user double-clicks on an } \\
\text { aircraft in BaseStation. }\end{array}$ \\
\hline
\end{tabular}

The transmission message is divided into eight sub-types and may be one of the following, see Table 2.

Table 2. MSG Sub Messages (Woodside, 2013)

\begin{tabular}{|c|c|c|}
\hline ID & Type & Description \\
\hline MSG,1 & $\begin{array}{l}\text { ES Identification and } \\
\text { Category }\end{array}$ & Conveys aircraft's callsign \\
\hline MSG,2 & $\begin{array}{l}\text { ES Surface Position } \\
\text { Message }\end{array}$ & Triggered by nose gear squat switch. \\
\hline MSG,3 & $\begin{array}{l}\text { ES Airborne Position } \\
\text { Message }\end{array}$ & As it says. \\
\hline MSG,4 & $\begin{array}{l}\text { ES Airborne Velocity } \\
\text { Message }\end{array}$ & As it says. \\
\hline MSG,5 & $\begin{array}{l}\text { Surveillance Alt } \\
\text { Message }\end{array}$ & $\begin{array}{l}\text { Triggered by ground radar if aircraft has sent } \\
\text { before MSG, } 1,2,3,4 \text { or } 8 \text { signal. }\end{array}$ \\
\hline MSG,6 & $\begin{array}{l}\text { Surveillance ID } \\
\text { Message }\end{array}$ & $\begin{array}{l}\text { Triggered by ground radar if aircraft has sent } \\
\text { before MSG, } 1,2,3,4 \text { or } 8 \text { signal. }\end{array}$ \\
\hline MSG,7 & Air To Air Message & $\begin{array}{l}\text { Triggered from TCAS. Conveys altitude and } \\
\text { "Ground" flag. }\end{array}$ \\
\hline MSG,8 & All Call Reply & $\begin{array}{l}\text { Broadcast but also triggered by ground radar. } \\
\text { Conveys only "Ground" flag. }\end{array}$ \\
\hline
\end{tabular}

Each of the aforementioned message types and sub-types can contain up to twenty-two comma-separated fields. Table 3 presents these entities with the proviso that the first ten fields apply to each message type, except Field 2 predestined only for $M S G$ type. Fields from eleven to twenty-two appear only 
along with the Transmission Message (MSG), and carry mainly airborne information. First ten fields provide data coming from the surveillance system.

Table 3. Fields of Messages of Type MSG (Woodside, 2013)

\begin{tabular}{|c|c|c|}
\hline ID & Type & Description \\
\hline Field 1: & Message type & (MSG, STA, ID, AIR, SEL or CLK) \\
\hline Field 2: & Transmission Type & $\begin{array}{c}\text { MSG sub types } 1 \text { to } 8 \text {. Empty field for other } \\
\text { message types. }\end{array}$ \\
\hline Field 3: & Session ID & Database Session record number \\
\hline Field 4: & AircraftID & Database Aircraft record number \\
\hline Field 5: & HexIdent & Aircraft Mode S hexadecimal code \\
\hline Field 6: & FlightID & Database Flight record number \\
\hline Field 7: & Date message generated & As it says \\
\hline Field 8: & Time message generated & As it says \\
\hline Field 9: & Date message logged & As it says \\
\hline Field 10: & Time message logged & As it says \\
\hline Field 11: & Callsign & $\begin{array}{l}\text { An eight digit flight ID - can be flight } \\
\text { number or registration (or even nothing). }\end{array}$ \\
\hline Field 12: & Altitude & $\begin{array}{c}\text { Mode C altitude. Height relative to } \\
\text { 1013.2mb (Flight Level). Not height } \\
\text { AMSL.. }\end{array}$ \\
\hline Field 13: & GroundSpeed & Speed over ground (not indicated airspeed) \\
\hline Field 14: & Track & $\begin{array}{l}\text { Track of aircraft (not heading). Derived } \\
\text { from the velocity E/W and velocity N/S }\end{array}$ \\
\hline Field 15: & Latitude & $\begin{array}{l}\text { North and East positive. South and West } \\
\text { negative. }\end{array}$ \\
\hline Field 16: & Longitude & $\begin{array}{l}\text { North and East positive. South and West } \\
\text { negative. }\end{array}$ \\
\hline Field 17: & VerticalRate & $64 \mathrm{ft}$ resolution \\
\hline Field 18: & Squawk & Assigned Mode A squawk code. \\
\hline Field 19: & Alert (Squawk change) & Flag to indicate squawk has changed. \\
\hline Field 20: & Emergency & $\begin{array}{l}\text { Flag to indicate emergency code has been } \\
\text { set }\end{array}$ \\
\hline Field 21: & SPI (Ident) & $\begin{array}{c}\text { Flag to indicate transponder Ident has been } \\
\text { activated. }\end{array}$ \\
\hline Field 22: & IsOnGround & $\begin{array}{l}\text { Flag to indicate ground squat switch is } \\
\text { active }\end{array}$ \\
\hline
\end{tabular}

\section{Uniform Data Layer}

The large amount of professional and amateur ADS-B receivers located on most continents, covering significant amount of the airspace, led to the conclusion that this kind of crowd-processing may establish valuable and reliable source of the data if using common interface. In this paper, authors 
have proposed the Semantic Web approach as a solution to the data integration problem. However, currently there is no uniform layer of the ADS-B data presentation and interfaces over the Web. One of the most popular air traffic tracking applications using ADS-B is Flightradar24. Although it is widespread and useful, it doesn't provide common interface. Being a commercial solution, it doesn't allow third-party applications to reuse the data.

Authors of this paper perceive information reprocessing as an upcoming challenge, especially in terms of ongoing development of Semantic Web technologies.

\section{Concept of Semantic Web}

Nowadays, we face a flood of information in every field. Bratt (2006) argues that keyword searching is effective for traditional text based data, but it can't provide satisfactory results from images, audio and video resources. Traditional searching is also sensitive due to ambiguity of words. These problems as well as data integration are the key issues that Semantic Web successfully addresses. It is an extension to the current web, which allows machines to carry out searching and processing of knowledge. Moreover, it allows reasoning processes to be performed, which may lead to the creation of new facts based on relations between existing knowledge.

For example, imagine two simple relations: Adam is husband of Eva and Adam is brother of Jenny. Knowing this and using proper ontology, machines could create the new fact that Jenny is the sister-in-law of Eva.

Bratt (2006) concludes that the Semantic Web is a machine-readable web, where resources are described using well-defined standards such as: URI, XML, RDF. There are new concepts being developed, built on top of the above-mentioned ones. This constitutes Semantic Web specifics. For this reason it's often called layer cake or semantic stack. Fundamental standards as well as concepts being developed are shown in Figure 6. 
Figure 6. Semantic Stack

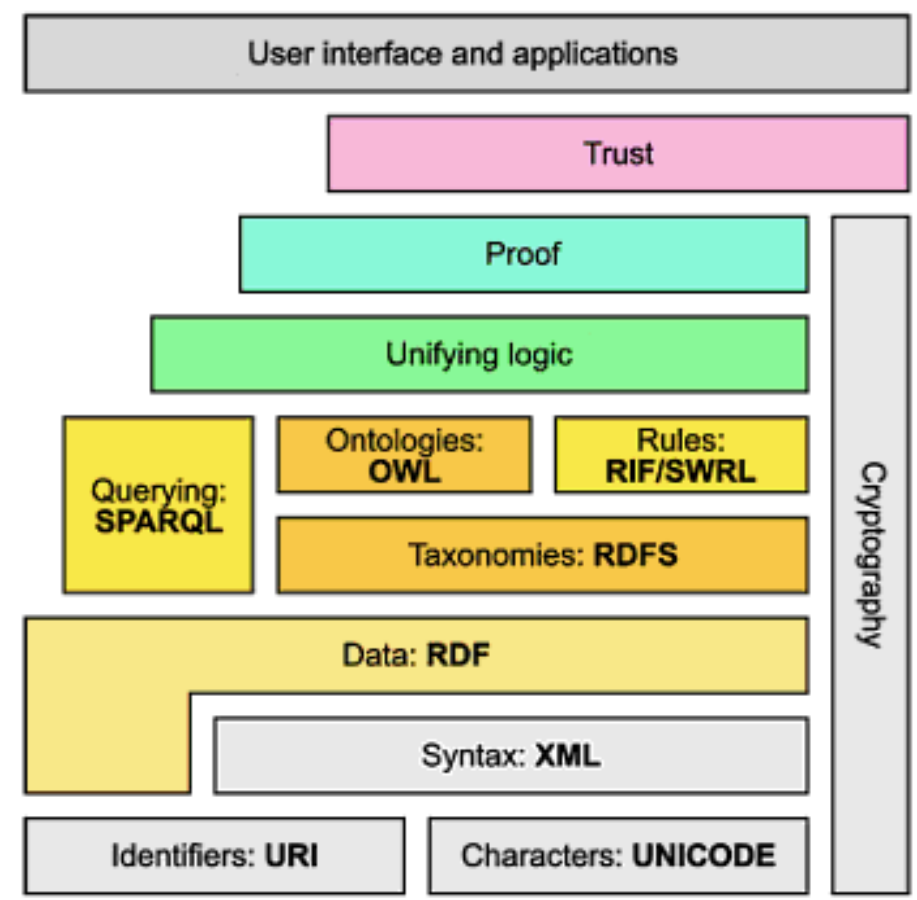

As Fensel, Hendler, Lieberman and Wahlster (2003) noted, HTML provides the proper display of the data, however it is not machine-readable. In other words, machines don't know the meaning of the data when they are parsing HTML markup; they only know how the information should be displayed.

For this reason eXtensible Markup Language (XML) was created. XML comes with special codes, called tags, which nested in documents give additional information about wrapped text. For example book can be

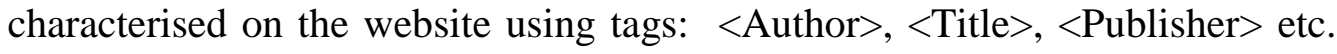
Programs can use these annotations in sophisticated ways, but a software developer must know what the website owner uses these tags for. In other words XML allows some structure to be built, but does not explain its meaning.

Berners-Lee, Hendler and Lassila (2001) state that the meaning can be provided by Resource Description Framework (RDF), using triples consisting of subject, predicate and object. Particular things (person, book, website) can have properties ("is spouse of", "has title") with literal values (another person, title, another website), vide previous example with Adam and Eva. Objects and subjects are expressed as Uniform Resource Identifiers (URIs). The most common type of URI is Uniform Resource Locator (URL) which is simply the web address (Berners-Lee, Hendler and Lassila 2001). One object can point to several subjects, where each of those subjects may be an object to another subject, creating a web (or a graph) of linked data. We distinguish different formats of RDF like Turtle, TriG, N-Triples, N-Quad or RDF/XML. Code 
snippet below is an example of how we could express this paper using machine-readable, RDF syntax.

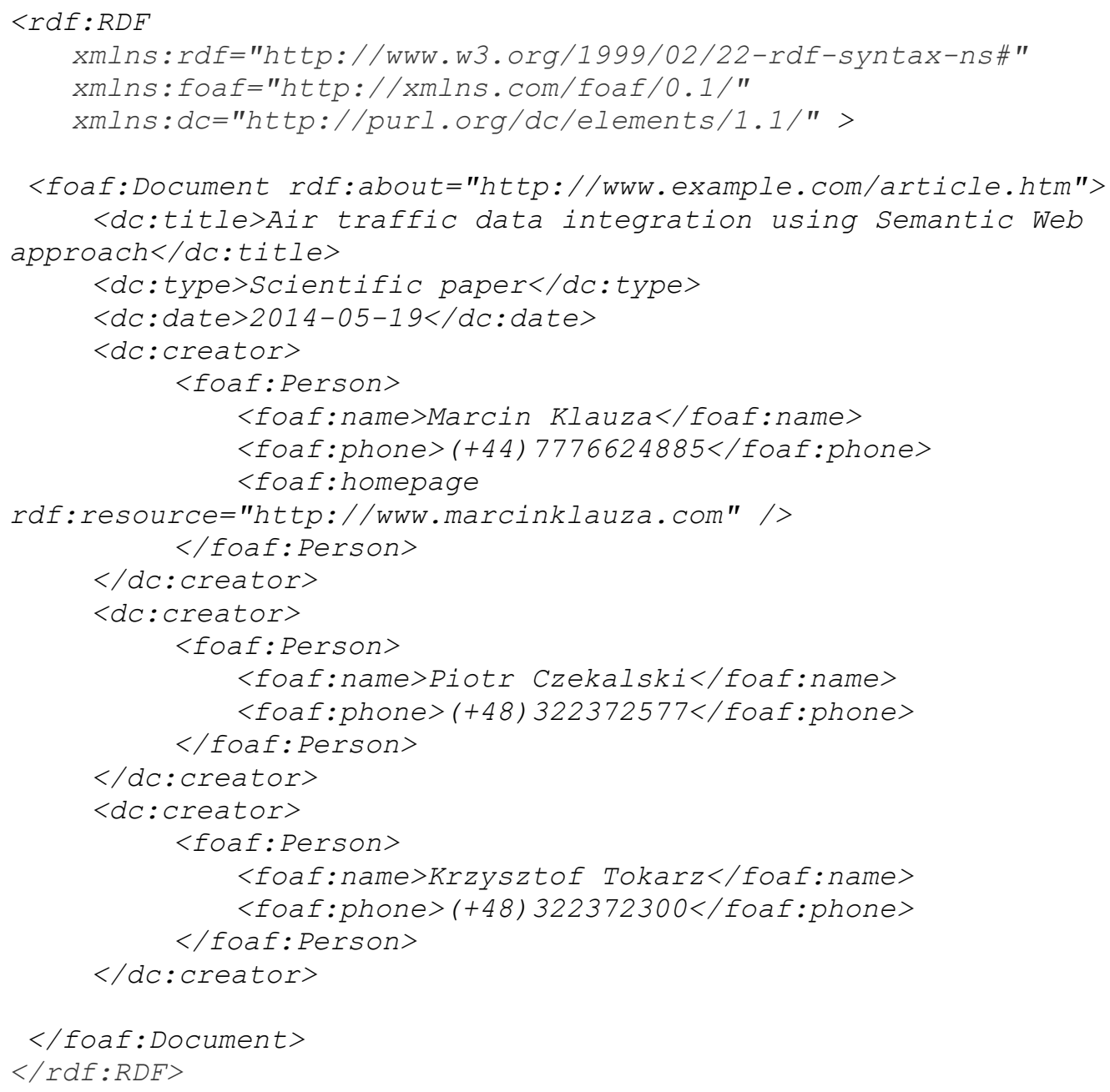

In the abovementioned example, the first tag comprises definitions of $r d f$, foaf and $d c$ namespaces. Foaf (an acronym for Friend of a friend) is an ontology describing people and their relations (Grimnes, Edwards and Preece 2004), whilst $d c$ (Dublin Core) is one of the most popular vocabulary set used for describing websites and physical artworks (books, CDs etc.) (Colomb, 2007). Words foaf and $d c$ are used thereafter instead of long URLs. Later in this example one can see the description of the document, its title, type, date, and authors. It is worth emphasising the semantics: Document is an object, type is a predicate and Scientific paper is a subject. Document also has a creator which is a person, having its own features.

\section{Web 3.0 Solution}

Software developed has been broken into two parts. First, Windows Service listening to 30003 port of SBS server, gathers and assembles data into the RDF repository. That collected data is published on the remote server, 
making it available for third-party applications. Finally web application residing in the server reads the RDF repository, checks whether some changes have been made and updates view in the client browser.

The Code snippet shown below is an example of aircraft description complying with RDF standard and using RDF/XML format.

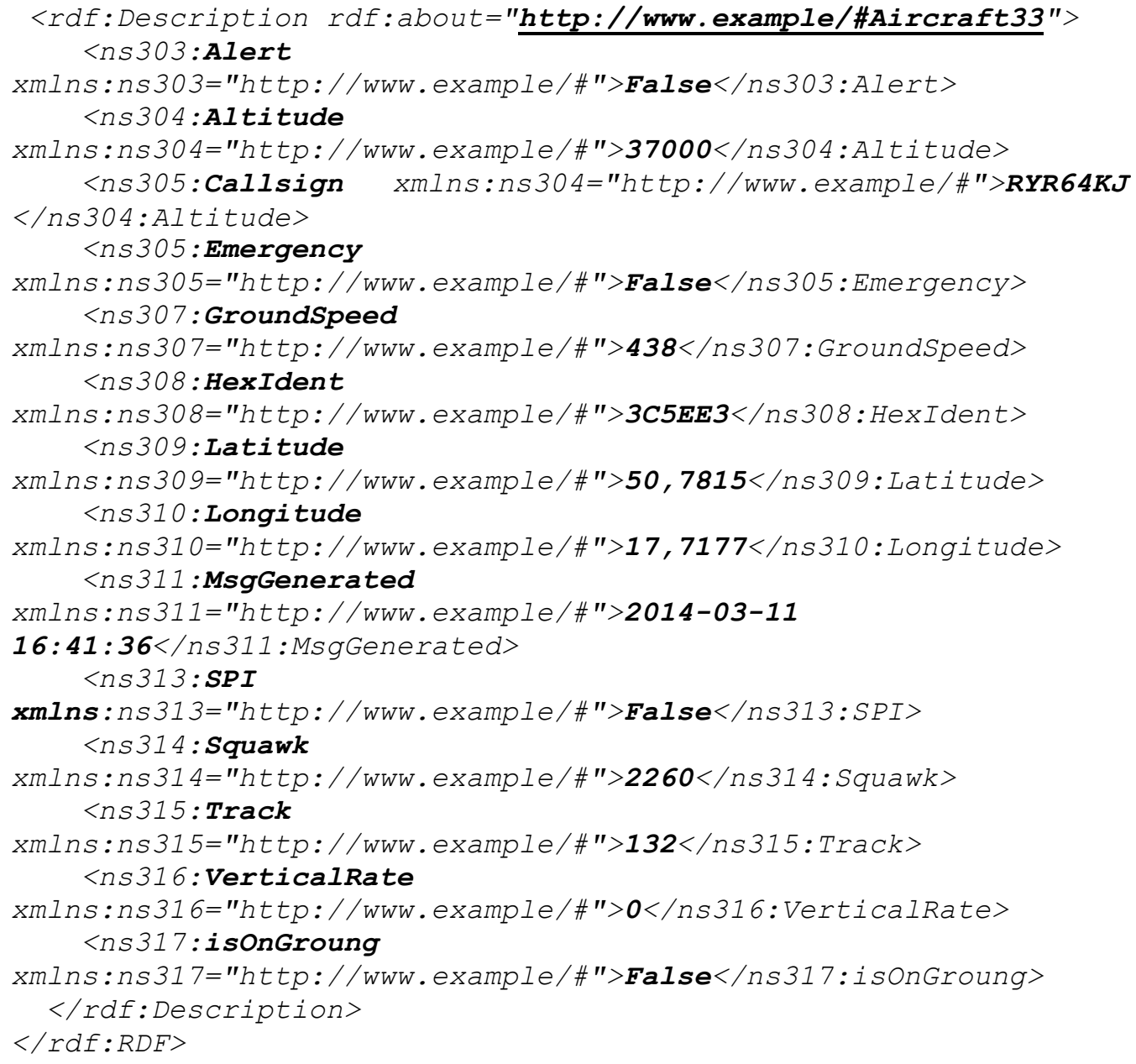

It has been generated using an open source C\#. Net library for RDF: dotNetRDF. This framework facilitates creating and searching in RDF graphs. In order to retrieve information from such graphs, there have been formulated queries in SPARQL (recursive acronym for SPARQL Protocol and RDF Query Language). It combines advantages of SQL-like language and the RDF data model. To list all the aircrafts along with their properties from the above-like repository, one could execute the following query: 
Vol. 2, No. 2 Klauza et al.: Air Traffic Data Integration using the Semantic Web Approach

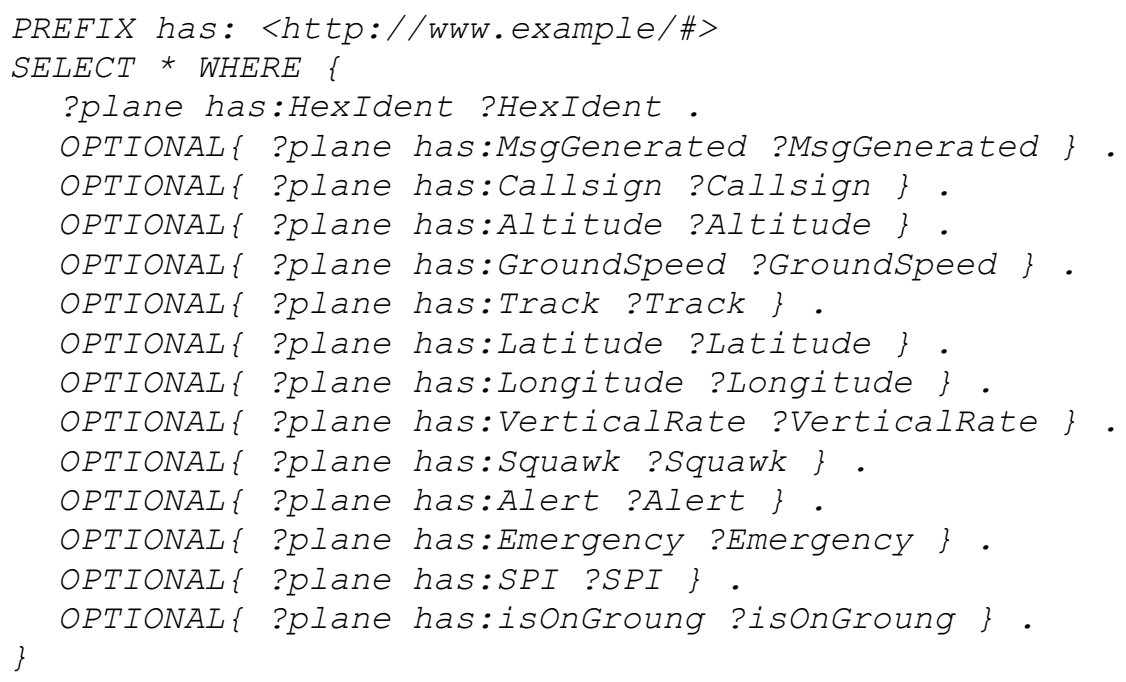

An exemplary website has been developed which queries RDF data set every few seconds and shows real-time information about the air traffic (Figure 7). This data comes from ADS-B receiver installed in the Silesian University of Technology; however this approach allows the integration of ADS-B data from different sources.

Figure 7. Exemplary Website utilising RDF Repository

\begin{tabular}{|c|c|c|c|c|c|c|c|c|c|c|c|c|c|c|c|}
\hline \multicolumn{14}{|c|}{ ADS-B radar Silesian University of Technology } & \multicolumn{2}{|c|}{ [ Log On ] } \\
\hline \multicolumn{16}{|c|}{$\begin{array}{l}\text { Last update: 2014-04-20 20:46:55 } \\
\text { Count: } 99 \\
\text { Status: OK }\end{array}$} \\
\hline No & $\begin{array}{l}\text { Mode S } \\
\text { hexadecimal } \\
\text { code }\end{array}$ & $\begin{array}{l}\text { Flight } \\
\text { ID }\end{array}$ & $\begin{array}{l}\text { Message } \\
\text { generated }\end{array}$ & Callsign & Altitude & GroundSpeed & $\begin{array}{l}\text { Track of } \\
\text { aircraft }\end{array}$ & Latitude & Longitude & $\begin{array}{l}\text { Vertical } \\
\text { rate }\end{array}$ & $\begin{array}{l}\text { Squawk } \\
\text { code }\end{array}$ & Alert & Emergency & SPI & isOnGroung \\
\hline 1 & $400 \mathrm{~B} 26$ & 1 & $\begin{array}{l}2014-04-20 \\
20: 46: 44\end{array}$ & AUA273 & 28850 & 443 & 322 & 48,4822 & 17,9726 & 960 & 2636 & False & False & False & False \\
\hline 2 & $4 \mathrm{BBC} 4 \mathrm{~B}$ & 0 & $\begin{array}{l}2014-04-20 \\
20: 46: 43\end{array}$ & OHY6524 & 34950 & 428 & 155 & 51,2145 & 17,0618 & 0 & 4710 & False & False & False & False \\
\hline 3 & 4BABEB & 10 & $\begin{array}{l}2014-04-20 \\
20: 46: 43\end{array}$ & THY1SG & 38000 & 458 & 293 & 49,2748 & 16,3569 & 0 & 3274 & False & False & False & False \\
\hline 4 & $471 F 51$ & 11 & $\begin{array}{l}2014-04-20 \\
20: 46: 43\end{array}$ & THY1ET & 35975 & 450 & 308 & 47,9702 & 19,4726 & 64 & 5404 & False & False & False & False \\
\hline 5 & 489526 & 14 & $\begin{array}{l}2014-04-20 \\
20: 46: 23\end{array}$ & AUA273 & 37000 & 450 & 231 & 50,6645 & 15,3135 & 0 & 5121 & False & False & False & False \\
\hline 6 & 489856 & 13 & $\begin{array}{l}2014-04-20 \\
20: 46: 43\end{array}$ & FHY346 & 34975 & 441 & 144 & 50,5674 & 19,6686 & -64 & 4646 & False & False & False & False \\
\hline 7 & 895057 & 18 & $\begin{array}{l}2014-04-20 \\
20: 46: 43\end{array}$ & RBA98 & 39000 & 493 & 117 & 49,3898 & 17,3454 & 0 & 372 & False & False & False & False \\
\hline 8 & 490116 & 2 & $\begin{array}{l}\text { 2014-04-20 } \\
20: 46: 43\end{array}$ & SUA703 & 41000 & 460 & 293 & 49,5752 & 15,2028 & 64 & 1464 & False & False & False & False \\
\hline 9 & $48 C B 15$ & 12 & $\begin{array}{l}2014-04-20 \\
20: 46: 31\end{array}$ & RYR65SN & 40000 & 450 & 39 & 49,1241 & 15,3735 & 64 & 2706 & False & False & False & False \\
\hline 10 & $3 \mathrm{C} 6 \mathrm{DC} 7$ & 22 & $\begin{array}{l}\text { 2014-04-20 } \\
20: 46: 40\end{array}$ & RYR45YH & 35650 & 462 & 340 & 52,0562 & 19,4762 & 0 & 1460 & False & False & False & False \\
\hline 11 & 4D208A & 20 & $\begin{array}{l}2014-04-20 \\
20: 46: 43\end{array}$ & VЛт671 & 45000 & 496 & 243 & 51,1367 & 16,6793 & 128 & 6252 & False & False & False & False \\
\hline 12 & 4CA762 & 24 & $\begin{array}{l}\text { 2014-04-20 } \\
20: 46: 43\end{array}$ & RYR8503 & 5700 & 213 & 165 & 50,1764 & 19,5044 & -640 & 3712 & False & False & False & False \\
\hline 13 & 3C4861 & 23 & $\begin{array}{l}2014-04-20 \\
20: 46: 42\end{array}$ & BER2355 & 36000 & 445 & 291 & 49,5521 & 15,0892 & 0 & 3206 & False & False & False & False \\
\hline 14 & $471 F 4 F$ & 15 & $\begin{array}{l}\text { 2014-04-20 } \\
20: 46: 27\end{array}$ & WZZ3WG & 36025 & 462 & 52 & 48,3666 & 17,6212 & 128 & 7622 & False & False & False & False \\
\hline 15 & 48AD09 & 28 & $\underset{\substack{2014-04-20 \\
21 . \wedge<.>A}}{2}$ & WZZ926 & 39000 & 451 & 308 & 47,6403 & 20,0947 & 0 & 5647 & False & False & False & False \\
\hline
\end{tabular}




\section{Conclusions}

Work described in this paper is a basis for the common interface for ADSB airborne data. Although proposed uniform data layer is a step forward, proper ontology has to be developed, so we can use the full potential of Semantic Web in aviation. In the near future authors would need to address this challenge.

\section{References}

Balara, D. (2010) Coaxial Collinear Antenna for ADS-B Receiver. [Online] Available from: http://www.balarad.net/. [Accessed: $10^{\text {th }}$ January 2014].

Berners-Lee, T., Hendler, J., and Lassila, O. (2001) The Semantic Web. Scientific American. 284(5). p. 34-43.

Bratt, S. (2006) Semantic Web and Other W3C Technologies to Watch. [Online] Available from: http://www.w3.org/2006/Talks/1023-sb-W3CTechSemWeb/W3 CTechSemWeb.pdf. [Accessed: $9^{\text {th }}$ April 2014].

Colomb, R.M. (2007) Ontology and the Semantic Web. Proceedings of the 2007 conference on Ontology and the Semantic Web. p.128-129.

Fensel, D., Hendler, J., Lieberman, H., Wahlster, W. (eds.) (2003): Spinning the Semantic Web. Bringing the World Wide Web to Its Full Potential. Cambridge: MIT Press. p. 1-20.

Grimnes, G.A., Edwards P., Preece A. (2004) Learning Meta-descriptions of the FOAF Network. The Semantic Web - Iswc. p.152-165.

Sprurt (2010) ADS-B receiver. ADS-B Decoder und Software mit PIC18F2550. [Online] Available from: http://www.sprut.de/electronic/pic/projekte/adsb/adsb. htm. [Accessed: 10 ${ }^{\text {th }}$ January 2014].

Touil, Y. (2012) ADSB\# - A simple and cheap ADS-B receiver using RTL-SDR. [Online] Available from: http://sdrsharp.com/index.php/a-simple-and-cheap-adsb-receiver-using-rtl-sdr. [Accessed: $10^{\text {th }}$ January 2014].

Ward, S. H., N0AX, ed. (2011). Chapter 22: Component Data and References. In The ARRL Handbook For Radio Communications (88th ed.). ARRL. p.22.

Woodside, J. (2013) SBS BaseStation, Article 4.2: Socket Data. [Online] Available from: http://www.homepages.mcb.net/bones/SBS/Article/Barebones42_Socket_ Data.htm. [Accessed: 25 ${ }^{\text {th }}$ March 2014]. 
